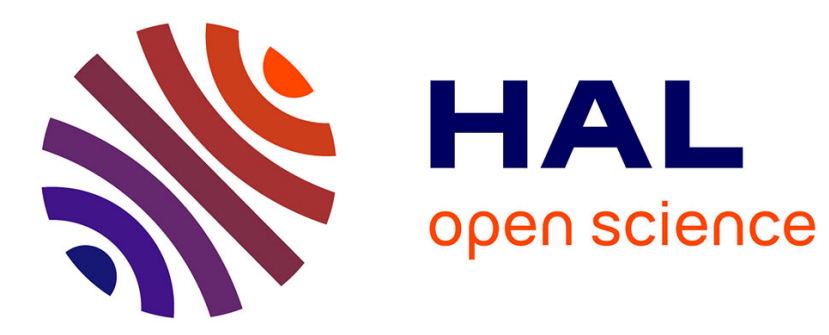

\title{
Optical pumping of helium-3 with a frequency electromodulated laser
}

\author{
M. Elbel, C. Larat, P.J. Nacher, M. Leduc
}

\section{To cite this version:}

M. Elbel, C. Larat, P.J. Nacher, M. Leduc. Optical pumping of helium-3 with a frequency electromodulated laser. Journal de Physique, 1990, 51 (1), pp.39-46. 10.1051/jphys:0199000510103900 . jpa-00212351

\section{HAL Id: jpa-00212351 https://hal.science/jpa-00212351}

Submitted on 1 Jan 1990

HAL is a multi-disciplinary open access archive for the deposit and dissemination of scientific research documents, whether they are published or not. The documents may come from teaching and research institutions in France or abroad, or from public or private research centers.
L'archive ouverte pluridisciplinaire HAL, est destinée au dépôt et à la diffusion de documents scientifiques de niveau recherche, publiés ou non, émanant des établissements d'enseignement et de recherche français ou étrangers, des laboratoires publics ou privés. 
Classification

Physics Abstracts

$34.40-42.60$

\title{
Optical pumping of helium-3 with a frequency electromodulated
} laser

\author{
M. Elbel $\left({ }^{1}\right)$, C. Larat $\left({ }^{2}\right)$, P.J. Nacher $\left({ }^{2}\right)$ and M. Leduc $\left({ }^{2}\right)$ \\ $\left({ }^{1}\right)$ Fachbereich Physik, Philipps Universităt Marburg, Renthof 5, D355 Marburg 1, F.R.G. \\ $\left({ }^{2}\right)$ Laboratoire de Spectroscopie Hertzienne de l'École Normale Supérieure, 24 rue Lhomond, 75231 \\ Paris Cedex 05, France
}

(Reçu le 23 juin 1989, accepté le 28 septembre 1989)

\begin{abstract}
Résumé. - Nous avons ajouté des bandes latérales à la structure de modes du laser utilisé pour le pompage optique d'un gaz d'hélium-3 au moyen d'un modulateur électro-optique externe. Nous avons ainsi montré que l'efficacité du processus de pompage optique peut être améliorée par une meilleure répartition de la puissance du laser sur le profil d'absorption Doppler des atomes d'hélium. Cette amélioration peut être expliquée par l'effet des collisions purement élastiques dans le gaz. Cette interprétation est confirmée par le résultat d'une expérience de pompage optique sélectif en vitesse.
\end{abstract}

\begin{abstract}
We have operated an electrooptical modulator to generate sidebands to the laser used for optical pumping of a helium-3 gas. Spreading the laser power over the Doppler absorption profile of the metastable helium atoms can enhance the efficiency of the optical pumping process. This improvement is discussed in terms of velocity changing collisions and compared to the result of a velocity selective optical pumping experiment.
\end{abstract}

\section{Introduction.}

Optical pumping is a powerful technique used for creating electronic or nuclear spin polarisation in a gas or a beam of atoms [1]. It results from the interactions between the atoms and a resonant light source, usually a tunable laser. When one deals with a gas, the atoms have a Maxwellian velocity distribution corresponding to an absorption frequency profile which can be much broader than the linewidth of the laser. As a result, only a small fraction of the atoms are directely excited by the laser. Actually, at high enough pressure, velocity changing collisions are likely to bring different atoms into resonance with the laser frequency, thus increasing the number of atoms effectively excited by the pumping light. Such collisional processes have been studied in detail, for instance in the case of krypton metastable atoms [2]. At usual pressures, they are not efficient enough to allow pumping of the whole velocity distribution of a gas.

This feature is considered in the present article, in the context of optical pumping of helium-3. A motivation for this work is the growing number of domains in which highly polarised helium-3 is 
required, such as the field of quantum fluids [3], polarised targets in nuclear physics [4], and helium spin filters for neutron beams [5].

Optical polarisation is obtained through a two-step process. It relies on optical pumping of the $2^{3} S_{1}$ metastable state of helium; metastability exchange collisions ensure the transfer of nuclear polarisation to the ground state atoms [6]. In order to excite a number of metastable atoms as large as possible with a laser, one can first rely on the above mentionned collisional processes. However, optimal conditions for creating metastable atoms limit the operating pressure to a few torr. Another possibility is to increase the number of longitudinal laser modes within the Doppler absorption profile of the gas, so as to excite more atoms in different velocity classes. Preliminary experiments have shown that a multimode laser polarises helium more efficiently than a singlemode one [7], especially at high laser power. This result is fairly well interpreted by the model derived in [7] for the kinetics of optical pumping.

In the present article, we report the results of an experiment in which the number of metastable velocity classes excited by the laser was increased using an electrooptical modulator outside the laser cavity to generate sidebands to each longitudinal mode. This set-up produced an enhanced efficiency of the helium-3 optical pumping, depending on the spacing of the sidebands. This result is interpreted in terms of collisional redistribution of atomic velocities. It is consistent with other results obtained in a velocity selective optical pumping experiment, which allowed measuring correlations between velocity and polarisation created by a singlemode laser. The latter experiment is also briefly described in this article.

\section{Modulating the frequency of a LNA laser:}

Optical pumping of helium-3 uses laser light at $1.083 \mu \mathrm{m}$, corresponding to the $2^{3} \mathrm{~S} \longrightarrow 2^{3} \mathrm{P}$ transition. A tunable laser at this wavelength was first obtained using NaF crystals coloured with $\left(\mathrm{F}_{2}^{+}\right)^{*}$ centers [8]; a singlemode ring version of this laser was built [9] and was used in the experiment described in paragraph 5. More recentely, a neodymium doped solid-state material, called LNA $\left(\mathrm{La}_{1-x} \mathrm{Nd}_{x} \mathrm{Mg} \mathrm{Al}_{11} \mathrm{O}_{19}\right)$, proved to be tunable around the required wavelength, and to polarise helium-3 nuclei up to $60 \%$ in a two-mode linear version [10]. The latter laser was used for the sideband generation. It was pumped by an ion argon laser, and included selective elements to ensure both tuning and operation on two consecutive longitudinal modes, $250 \mathrm{MHz}$ apart. The output beam was first passed through a telescope, which increased its diameter by a factor of 3 , but decreased its divergence. It was then focused through the electrooptical modulator by a lens of $30 \mathrm{~cm}$ focal length.

This electrooptical modulator (EOM) consisted of a lithium tantalate crystal of dimensions $0.65 \times$ $0.50 \times 25.0 \mathrm{~mm}^{3}$. The laser beam was passed along the long axis which was identical with the $y$ axis in Yariv's nomenclature [11]. The $z$ axis was directed along the height $(0.65 \mathrm{~mm})$, perpendicular to the polarisation of the laser. The crystal base was glued to a copper bed with a $1 \mathrm{~mm}$ indium inlay. The glue was electrically conducting. On top, a fine gold wire was attached by the same glue, which was spread to cover the whole surface. This EOM crystal had been previously used at the wavelength $\lambda=596 \mathrm{~nm}$ for which its faces were antireflection coated. At the wavelength $\lambda=$ $1083 \mathrm{~nm}$, this non adequate coating caused a $30 \%$ loss on the transmitted power.

At $596 \mathrm{~nm}, 10 \mathrm{~W}$ of radiofrequency power produced a large modulation of the laser mode, with complete disappearance of the carrier [12]. In this case, the EOM was just used as a piece of a $50 \Omega$ cable. This simple set-up was not appropriate here, as a similar modulation at $\lambda=1083 \mathrm{~nm}$ requires more radiofrequency power. Instead the EOM was used as a part of a resonating circuit, as shown in figure 1. For frequencies below $175 \mathrm{MHz}$, tank circuits were suitable: figure 1a shows the corresponding set-up. For higher frequencies, the EOM crystal was used as a part of a $\lambda / 4$ resonator. Eigen frequencies up to $400 \mathrm{MHz}$ could be obtained with the set-up shown in figure $1 \mathrm{~b}$. 
Figure 2 displays a sequence of spectra observed with a confocal Fabry Perot analyser. The upper trace shows the double mode structure of the LNA laser in the absence of modulation. The lower traces correspond to increasing values of the radiofrequency power applied to the EOM. Two sidebands on both sides of each carrier mode could thus be easily produced.

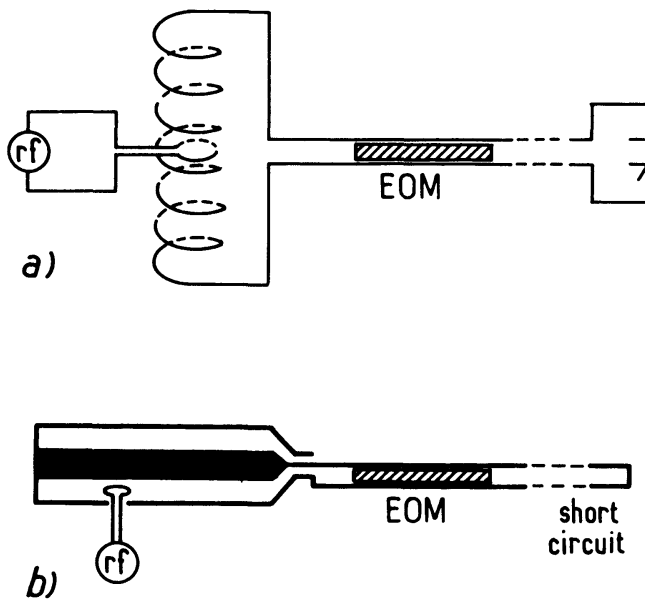

Fig. 1.

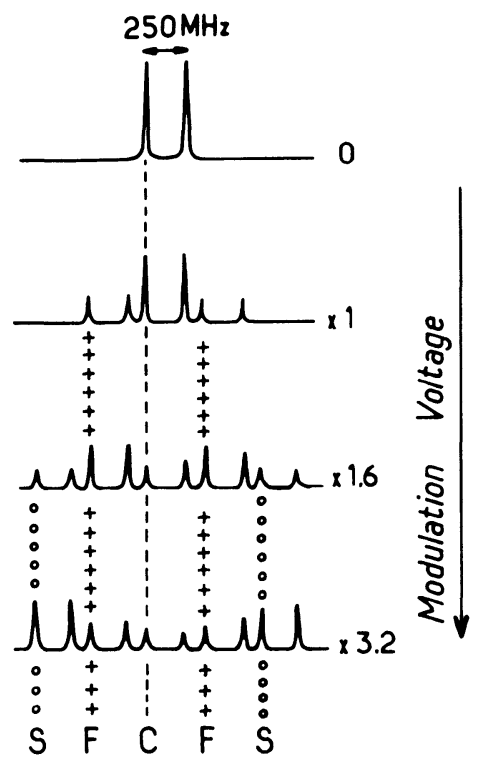

Fig. 2.

Fig. 1. - Two resonator circuits used to create sidebands to the laser modes with the EOM crystal. a) Tank circuit used for frequencies up to $175 \mathrm{MHz}$. b) $\lambda / 4$ resonator used for higher frequencies, the EOM being part of a $50 \Omega$ wave guide. The dimensions of the resonator are: $75 \mathrm{~mm}$ in length, $25 \mathrm{~mm}$ and $10 \mathrm{~mm}$ for the external and internal diameters respectively. The eigenfrequency is about $200 \mathrm{MHz}$, or $400 \mathrm{MHz}$ when the end of the EOM is short circuited to make a $\lambda / 2$ resonator.

Fig. 2.- Mode structure of the modulated laser light. The sprectra were obtained using a piezo-scanned FabryPerot analyser. The upper trace was obtained without modulation. The lower traces were obtained with increasing values of the modulation power. The modulation frequency was $370 \mathrm{MHz}$. For each of the laser modes, as the first and second sidebands ( $F$ and $S$ ) grow with the radiofrequency power, the amplitude of the carrier (C) decreases.

\section{Polarising helium-3 with the modulated LNA laser:}

The laser source described above was used in an optical pumping experiment. The helium-3 cell was a cylinder $(5 \mathrm{~cm}$ in diameter, $5 \mathrm{~cm}$ in length) filled at a pressure of 0.3 torr and held in a homogeneous magnetic field of 6 Gauss. The laser beam was expanded to cover a large part of the cell area, circularly polarised with a quarter-wave plate, passed through the cell along the field axis and reflected back into the cell by a metallic mirror. The laser frequency was tuned on the $\mathrm{C}_{8}$ component of the absorption line $\left(2^{3} S_{1}, F=1 / 2 \longrightarrow 22^{3} \mathrm{P}_{0}\right.$ transition), which is best suited for pumping at high laser power [7]. The nuclear polarisation of the atoms was monitored by observing 
the circular polarisation of the line at $\lambda=668 \mathrm{~nm}$ emitted by the discharge, according to the method described in [13].

Figure 3 shows the time build-up of the nuclear polarisation when the laser is applied to the cell. At first there was no modulation of the laser frequency. The polarisation amounted to $50 \%$, as can be expected for a two-mode laser of $250 \mathrm{~mW}$ power [10]. The modulating radiofrequency was then switched on, at a frequency of $175 \mathrm{MHz}$, and with an amplitude corresponding to the generation of sidebands on each side of each carrier, as shown by the lowest trace in figure 2. The nuclear polarisation consequentely increased, reaching a new equilibrium value after a time comparable to that of the initial build-up. The radiofrequency was then switched off and the polarisation decreased back to its original value. A relative enhancement of about $8 \%$ of the nuclear polarisation resulted from the spreading of the laser power over a larger number of modes.

The same experiment was repeated for various sideband spacings, generated using the devices shown in figure 1 . The amplitude of the modulation was adjusted to keep the ratio of carrier to sideband amplitudes constant. The relative increase in nuclear polarisation resulting from the modulation is shown in figure 4 as a function of the frequency of modulation. Above $50 \mathrm{MHz}$, the observed effect does not show any significant dependance on the frequency of the modulating voltage. This could be expected since the frequency spreading of the modulated beam remained within the absorption profile in all cases. Conversely, for the same estimated sideband amplitude, the enhancement was smaller when the frequency of the modulation was below $20 \mathrm{MHz}$. However, due to the limited resolution of our spectrum analyser, it was not possible to measure the relative amplitudes of the carrier and sidebands, and we did not get quantitative data to plot in figure 4.

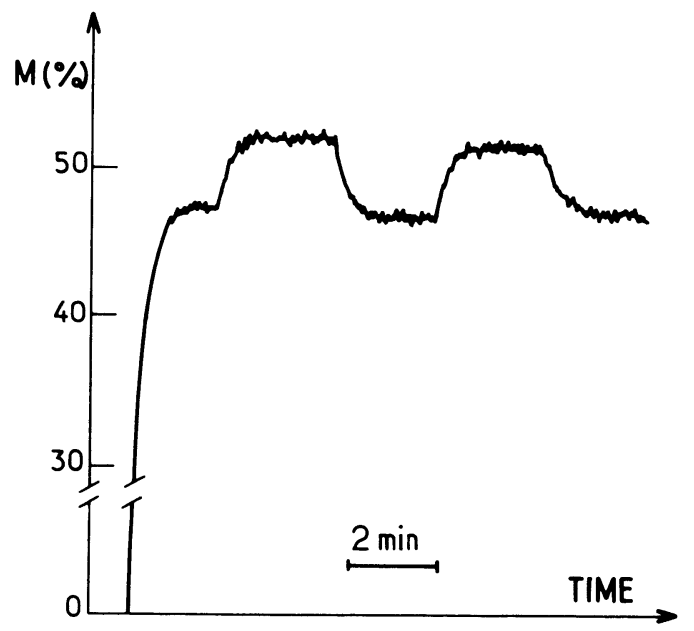

Fig. 3.

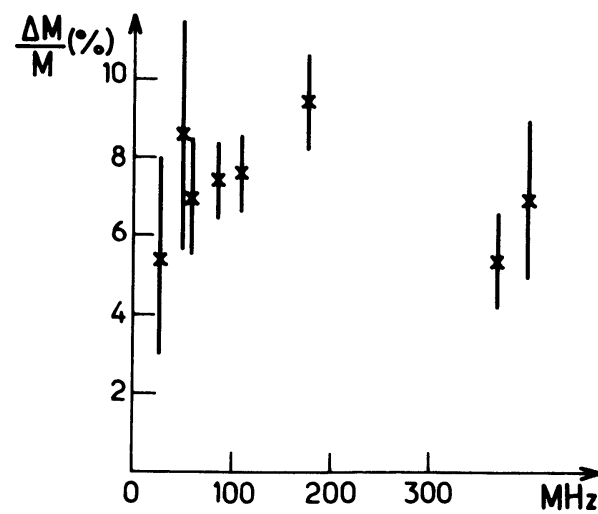

Fig. 4.

Fig. 3.- Time evolution of ${ }^{3} \mathrm{He}$ nuclear polarisation $M$. It first grows from zero to its asymptotic value when the unmodulated pumping light is applied. When the radiofrequency is switched on, the polarisation increases. After modulation has been turned off, $M$ comes back to its initial value. The experiment was repeated twice.

Fig. 4.- Relative variation of ${ }^{3} \mathrm{He}$ nuclear polarisation $M$ when the modulation is switched on, as a function of the modulating frequency. No significant frequency dependance of the polarisation increase is observed above $50 \mathrm{MHz}$ (see text). 


\section{Discussion.}

The velocity dependance of the laser excitation tends to create correlations between atomic velocities and internal atomic variables, whereas collisions constantly tend to destroy these correlations. The orientation of each velocity class of metastable $2^{3} \mathrm{~S}$ atoms thus results from several competing processes, and so does the nuclear polarisation transferred to the ground state. The model developped in [7] simply assumes that a fraction $n_{\mathrm{m}}^{*} / n_{\mathrm{m}}$ of the atoms in the metastable state is effectively interacting with the pumping light. Figure 5 shows the computed equilibrium polarisations as a function of the laser power for different values of that fraction, as well as the results of the experiment described above.

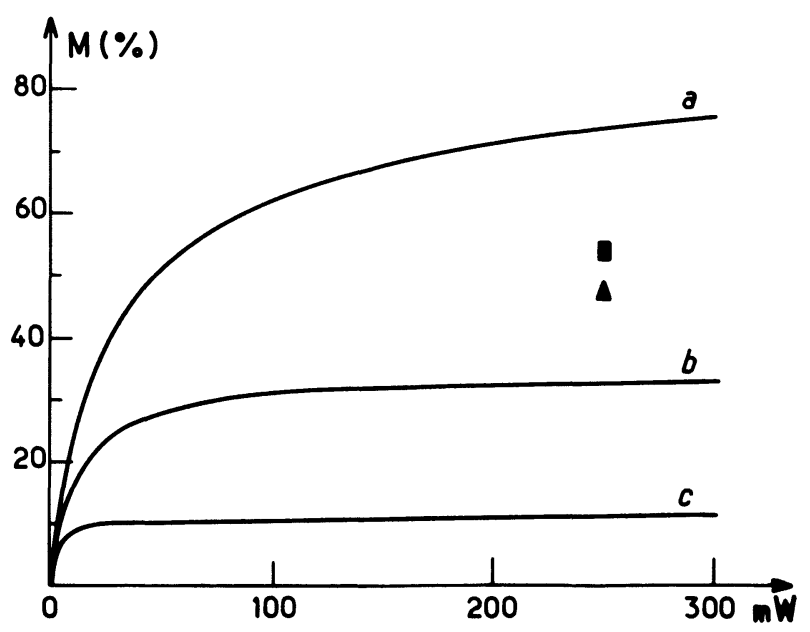

Fig. 5.- Computed equilibrium polarisation $M$ as a function of the laser power. a) Ideal broadband pumping, matching the whole Doppler absorption profile $\left(n_{\mathrm{m}}^{*} / n_{\mathrm{m}}=1\right)$. b) Singlemode laser pumping, taking into account velocity changing collisions $\left(n_{\mathrm{m}}^{*} / n_{\mathrm{m}}=1 / 120\right)$ c) Singlemode laser pumping, assuming no velocity changing collisions occur $\left(n_{\mathrm{m}}^{*} / n_{\mathrm{m}}=1 / 600\right)$. The data corresponding to the present experiment are also shown ( $\Delta$ : without modulation; $\square$ :with modulation).

Curve (a) is for $n_{\mathrm{m}}^{*} / n_{\mathrm{m}}=1$, corresponding to an ideal situation where a broadband pumping light would just be matching the Doppler absorption profile. Curve (c) would correspond to a monochromatic pumping light in the absence of velocity changing collisions; the value of $n_{\mathrm{m}}^{*} / n_{\mathrm{m}}$ is set equal to the ratio between the linewidth associated to the excited $2^{3} \mathrm{P}$ state lifetime $(3.3 \mathrm{MHz})$, and the Doppler linewidth $(2 \mathrm{Ghz})$. Curve (b) is for $n_{\mathrm{m}}^{*} / n_{\mathrm{m}}$ set to five times the previous value, and was found in [7] to fit the experimental results for monochromatic (single mode laser) pumping in presence of velocity changing collisions for a pressure of 0.3 Torr. As expected, the results of the present experiment lie between curves (a) and (b), since the laser had more than one longitudinal mode. The polarisation obtained when modulating lies above that obtained without modulation: the larger the number of laser modes within the Doppler profile, the higher the value of the fraction $n_{\mathrm{m}}^{*} / n_{\mathrm{m}}$ of effectively pumped atoms.

The difference between the calculated curves can be qualitatively understood. At low pumping power and low polarisation, optical pumping is a linear process and no saturation of the transi- 
tion occurs: the effectively pumped fraction $n_{\mathrm{m}}^{*} / n_{\mathrm{m}}$ is irrelevant to the result, and all curves have the same slope at the origin. On the other hand, at high laser power, it is easier to saturate the transition and be limited to low nuclear polarisation if that fraction is small, due to monochromatic pumping and/or poor collisional velocity changes. As a consequence, sidebands generation increases the efficiency of the optical pumping process as soon as they increase the effectively pumped fraction $n_{\mathrm{m}}^{*} / n_{\mathrm{m}}$, that is as soon as they differ in frequency from the carrier by more than the width associated with collisional redistribution. For a pressure of 0.3 Torr, that width can be directely measured (see below), or estimated from the value of $n_{\mathrm{m}}^{*} / n_{\mathrm{m}}$ used for curve (b) which best fits experimental results for single mode pumping. Both methods lead to a value of the order of $20 \mathrm{MHz}$ for the collisional width, which is consistent with the frequency dependance of the polarisation enhancements obtained in the previous section. That width can be attributed to velocity changing collisions, as it is much larger than the natural linewidth (the saturation broadening is negligible when using an expanded beam).

\section{Velocity selective optical pumping in helium-3.}

Velocity selective optical pumping is a method to measure correlations between velocity and polarisation of the metastable atoms. It was first demonstrated with metastable neon atoms [14]. Our experimental set-up is shown in figure 6. The light source was the singlemode ring colour

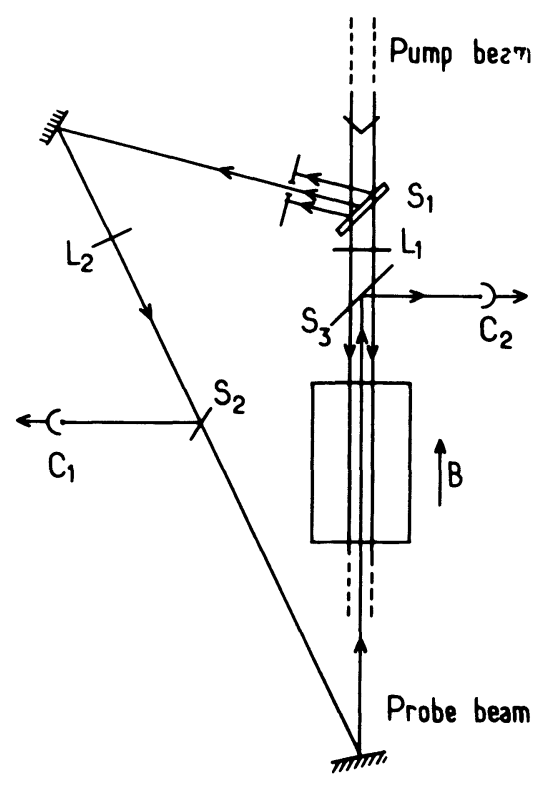

Fig. 6.- Set-up of the velocity selective optical pumping experiment. The pump beam, parallel to the magnetic field $B$,is circularly polarised by the quarter-wave plate $\mathrm{L}_{1}$. It polarises ${ }^{3} \mathrm{He}$ nuclei in a cell where a discharge populates the pumped metastable state. The circular polarisation of the probe beam is periodically reversed, due to the rotation (at a frequency $\Omega$ ) of quarter-wave plate $\mathrm{L}_{2}$. The difference between signals recorded in $\mathrm{C}_{1}$ and $\mathrm{C}_{2}$ is proportional to the average absorption of the cell, and modulated at frequency $\Omega$. The resulting AC signal is phase detected, and proportional to the polarisation of the probed metastable atoms. $S_{1}, S_{2}$ and $S_{3}$ are beam splitters used to separate probe and pump beams. 
centre laser described in [9]. The pump beam was weak enough so that the nuclear polarisation was a linear function of the laser power. A small amount of power, deflected from the pump beam, became a counterpropagating probe beam. The pump beam was circularly $\sigma^{+}$polarised while the circular polarisation of the probe beam was periodically reversed. We measured the absorption of the probe beam, whose modulation was proportional to the polarisation of the metastable atoms. Figure 7 shows the recorded signal obtained by sweeping the laser frequency across the Doppler profile of the metastable atoms, slowly enough to keep the nuclear polarisation at its equilibrium value.
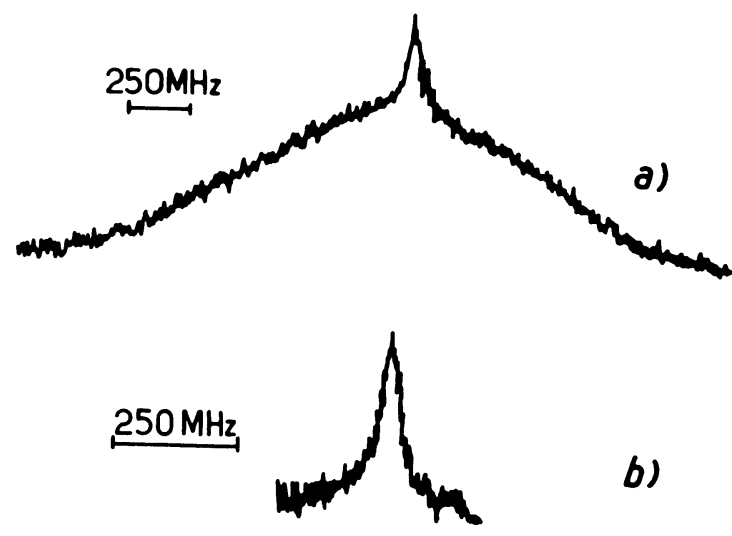

b)

Fig. 7.- Polarisation of ${ }^{3} \mathrm{He}$ metastable atoms as a function of laser frequency, recorded in the VSOP experiment shown in figure 6. a) All velocity classes of the atoms are explored b) Detail of the central peak. Its width is determined by velocity changing collisions (see text).

The pump and probe beams being counterpropagating, they interact with atoms which have opposite velocities along the laser direction: these atoms are different, except at the center of the profile where this velocity is zero. Outside the narrow central peak, one thus observes the polarisation of metastable atoms not interacting with the pump beam; their polarisation is in equilibrium with that of the ground state atoms through the metastability exchange collisions. The central narrow peak shows that atoms effectively interacting with the laser are overpolarised. The width of this central peak is roughly $20 \mathrm{MHz}$. It gives a value for the broadening of the velocity class effectively interacting with the laser due to velocity changing collisions. This value is consistent with the results obtained in section 3 , as discussed in section 4 .

\section{Conclusion.}

In practice, the use of the electrooptical modulator can increase the nuclear polarisation obtained by laser optical pumping of helium 3. The frequency of the generated sidebands is not critical and can range between 50 and $400 \mathrm{MHz}$. An increase of polarisation from $50 \%$ to $55 \%$ was observed at $280 \mathrm{~mW}$ of laser power. The increase is expected to be even more significant at higher laser level. 
Optimisation of the LNA laser and of the EOM crystal (for instance by appropriate anti-reflection coating) should lead to over $60 \%$ of nuclear polarisation. Our present results can be compared to the polarisation rates obtained in other optical pumping experiments performed with frequency modulated multimode dye lasers on argon metastable beams [15]. The main difference is that a gas exibits a much broader absorption profile than a beam. Since each laser mode interacts only with a narrow fraction of this profile, generating sidebands to the laser appears particularly well suited in the case of a gas such as helium 3.

Acknowledgements.

One of the authors (M.E.) received support from the Deutsche Forschungsgemeinschaft (DFG).

\section{References}

[1] See for instance:

KASTLER A., J. Phys. Rad. 11 (1950) 255.

HAPPER W., Rev. Mod. Phys. 14 (1972) 169.

[2] BRECHIGNAC C., VETTER R. and BERMAN P.R, Phys. Rev. A 17 (1978) 1609.

[3] LHUILlier C. and LALOE F., J. Phys. France 40 (1979) 239.

[4] Workshop on "Polarized helium-3 beams and targets", AIP proceedings 131 (New York) 1985.

[5] PASSEL L. and SCHERMER R.I., Phys. Rev 150 (1966) 146.

[6] COLEGROVE F.D., SCHEARER L.D. and WALTERS G.K, Phys. Rev. 132 (1963) 2561.

[7] NACHER P.J. and LEDUC M., J. Phys. France 46 (1985) 2057.

[8] MOLlENAUER L.F., Opt. Letters 5(1980) 188.

[9] TRÉNEC G., NACHER P.J. and LEDUC M., Opt. Commun. 43 (1982) 37.

[10] DANIELS J.M., SCHEARER L.D., LEDUC M. and NACHER P.J., J. Opt. Soc. Am. 4(1987) 1133.

[11] YARIV A and YEH P., "Optical waves in crystals: propagation and control of laser radiation", Eds. John Wiley \& Sons (New York) 1984.

[12] REICH H. and JANSCH H.J., to appear in Nuclear Instruments and Methods.

[13] PINARD M. and VAN DER LINDE J., Can. J. Phys. 52 (1974) 1615.

[14] PINARD M., AMINOFF C.G. and LALOE F., Phys. Rev: A 19 (1979) 2366.

[15] GIBERSON K. W., HAMMOND M.S., HART M.W., LYNN J.G. and DUNNING F.B., Opt. Lett. 10 (1985) 119. 